\title{
The benefit of fibrosa layer stripping technique during minimally invasive aortic valve replacement for calcified aortic valve stenosis - A randomized controlled trial
}

\author{
Yuan Zhou ${ }^{1}$, Liang Zhang ${ }^{1}$, Kun Hua ${ }^{1}$, Jinwei Zhang ${ }^{1}$, and Xiubin Yang $^{1}$ \\ ${ }^{1}$ Capital Medical University Affiliated Anzhen Hospital
}

July 31,2020

\begin{abstract}
Background: Fibrosa layer stripping (FLS) technique is a new approach to remove calcified aortic valve. In this study, we aimed to assess the effectiveness of the FLS technique by comparing with the conventional technique in minimally invasive aortic valve replacement (MIAVR). Methods: A prospective, single-center, randomized controlled trial was conducted at Beijing Anzhen Hospital. 70 patients diagnosed with severe calcific aortic stenosis were randomly assigned to undergo FLS (n=35) or conventional $(\mathrm{n}=35)$ technique to debride calcified aortic valve. Preoperative profile, procedural parameters, and postoperative outcomes were analyzed. Results: No significant difference was observed in the preoperative profile between two groups. Compared with the conventional technique, the FLS technique had significantly higher indexed effective orifice area and lower mean gradient. Moreover, FLS technique was associated with significantly reduced aortic cross-clamp time (41[38-44] vs 56[5160] min, $\mathrm{p}<0.001)$, cardiopulmonary bypass (CPB) time (63[56-69] vs 81[75-84] min, $\mathrm{p}<0.001)$ and operative time (148[141-156] vs $173[169-180]$ min, $\mathrm{p}<0.001)$. Lastly, the length of intensive care unit stay $(1.2 \pm 0.4$ vs $1.5 \pm 0.8$ days, $\mathrm{p}=0.041)$ and hospital stay ( $5.3 \pm 0.6$ vs $6.0 \pm 1.4$ days, $\mathrm{p}=0.020)$ was significantly reduced in the FLS group compared with those in the conventional group. Conclusions: FLS technique is effective in removing calcified tissue during MIAVR and is associated with shorter cross-clamp time and CPB time, and better hemodynamic performance than conventional technique.
\end{abstract}

\section{KEYWORDS:}

Fibrosa layer stripping; Calcific aortic stenosis; Minimally invasive aortic valve replacement.

\section{ABBREVIATIONS AND ACRONYMS}

AVR aortic valve replacement

AS aortic stenosis

MIAVR minimally invasive aortic valve replacement

CPB cardiopulmonary bypass

FLS fibrosa layer stripping

BSA body surface area

ICU intensive care unit

CT computer tomography

EOA effective orifice area

PVL paravalvular leak 
MRI magnetic resonance imaging

PPM prosthesis-patient mismatch

NYHA New York Heart Association

BNP brain natriuetic peptide

LVEF left ventricular ejection fraction

$\mathrm{AVC}_{\text {density }}$ aortic valve calcium density

AU agatston unit

COPD chronic obstructive pulmonary disease

\section{INTRODUCTION}

Aortic Valve Replacement (AVR) is by far the most effective approach to treat aortic stenosis (AS). Since the implementation of minimally invasive aortic valve replacement (MIAVR) by right thoracotomy in 1993, a variety of surgical methods including partial midline sternotomy, reverse sternotomy, parasternal approaches, and port-access via thoracoscopy have been developed. ${ }^{1-3}$ Compared with conventional AVR, MIAVR has several advantages, including reduced blood transfusion, less pain, excellent postoperative outcome, quicker recovery, and better cosmesis. ${ }^{4-5}$ Despite differences in surgical approach, the basic operative procedure has remained similar. Following aortotomy, the diseased valve leaflets are excised and the annulus is debrided. Then the prosthetic valve is anchored in the aortic annulus by sutures under cardiopulmonary bypass (CPB) and cross-clamping of the aorta. Removal of the severely calcified aortic valve and annulus is the most challenging step.

The conventional technique in MIAVR has several limitations. It mechanically pulverizes the calcified tissue on the valve and annulus, which requires prolonged cross-clamping time and CPB time. The residual tissue generated during annular debridement may cause perioperative myocardial infarction and cerebral infarction. Incomplete removal of calcification also prohibits implantation of a prosthetic valve of proper size and may cause perivalvular leakage. Recently, ultrasonic aspirator has been applied during MIAVR. ${ }^{6}$ However, in our practice, this method generates large amount of debris, is not effective in crushing calcified tissue deeply wrapped by the intimal tissue, and causes damage to the local normal tissues by ultrasonic energy. ${ }^{7}$

Aortic valve leaflet is composed of 4 clearly defined tissue layers: the endothelium, fibrosa, spongiosa and ventricularis. The valve leaflets are attached to aortic valve annulus which is a dense collagenous network. ${ }^{8}$ The aortic valve annulus is covered by fibrosa, which continues to cover the adjacent valve tissue. ${ }^{9}$ The calcific nodules and lesions tend to occur primarily in the fibrosa layer and extend to the aortic side of the valve. ${ }^{10}$ Therefore, calcified tissue is formed not only on the valve, but also on the annulus. As long as the fibrosa layer is peeled off, the purpose of removing calcification can be achieved. Histologically, the spongiosa layer is located below the fibrosa layer. The spongiosa layer, which has a high proteoglycan content, is a layer of loose connective, ${ }^{11}$ so it is easy to separate the fibrosa layer from the spongiosa layer. Taking advantage of the above pathological characteristics of calcified tissue, we have developed a new surgical technique, which we coined a term as fibrosa layer stripping (FLS), to peel off the fibrosa layer at the calcified tissue of aortic annular.

In order to evaluate the potential benefits of using FLS technique in removing the calcified tissue in MIAVR, we designed a prospective and randomized clinical trial and assessd its short- and long-term outcomes compared to conventional technique in present study.

\section{MATERIAL AND METHODS}

This prospective, single-center, randomized trial was registered with the Chinese Clinical Trial Registry as ChiCTR 2000031338 and conducted at Beijng Anzhen Hospital. All patients provided written informed 
consent. The trial was conducted in compliance with the International Conference on Harmonisation and the Declaration of Helsinki. This study was approved by the Beijing Anzhen Hospital Medical Ethics Committee.

\subsection{Study subjects}

Patient enrollment criteria: age [?]18 years old; diagnosed with severe calcific AS by echocardiography with a maximum aortic jet velocity $>4.0 \mathrm{~m} / \mathrm{s}$ and mean gradient $>40 \mathrm{~mm} \mathrm{Hg}$, or continuity equation valve area $<1.0 \mathrm{~cm}^{2}$, or valve area indexed for body surface area (BSA) $<0.6 \mathrm{~cm}^{2} / \mathrm{m}^{2} ;{ }^{12}$ considered for a biological aortic valve. Exclusion criteria included planned and unplanned concomitant procedure, history of previous cardiac surgery, thoracic deformity, porcelain ascending aorta, and high surgical risk if patient's Society of Thoracic Surgeons (STS) score was higher than $8 \%$.

\subsection{Study design}

70 patients who met the above criteria were enrolled between February 2016 and December 2018, with the last follow-up in December 2019. Patients were randomly assigned to the FLS group $(n=35)$ or the conventional group $(\mathrm{n}=35)$. Randomized numbers were generated by professional statisticians according to random digit table. 10 patients were excluded from the study for the following reasons: sudden death before operation $(\mathrm{n}=3)$, withdrawal of consent $(\mathrm{n}=5)$, admission to an intensive care unit (ICU) for mechanical ventilation $(n=1)$, and violation of protocol $(n=1)$. In addition, 2 patients in the FLS group and 1 patient in the conventional group underwent concomitant aortic annular enlargement due to a small aortic annulus mismatched with BSA. Therefore, 28 (28/30) patients in the FLS group and 29 (29/30) patients in the conventional group were included in per-protocol analysis.

\subsection{Surgical procedure}

The operations were performed by the same surgeon. Both groups of patients underwent MIAVR via an upper sternum approach. Briefly, an upper hemisternotomy to the right side was performed in the midline and the third or fourth intercostal space guided by the preoperative chest computer tomography (CT), forming a J-shape incision. The distal ascending aorta was cannulated. Multi-stage venous cannulation (Medtronic, Minneapolis, MN) was performed through the femoral vein. The ascending aorta was cross-clamped and crystalloid was perfused antegrade through the aortic root soon after initiating CPB, while letting the nasopharyngeal temperature drip to $32-34^{\circ} \mathrm{C}$. If the patient has combined aortic valve insufficiency, the crystalloid was perfused through the coronary artery sinus after the ascending aorta was incised. A transverse incision was made $1 \mathrm{~cm}$ above the sinotubular junction, and 3 traction sutures were drawn on the upper and lower edges of the incision to expose the aortic valve. In the FLS group, scissor was used to cut off as many diseased leaflets as possible, and a nerve hook was used to incise a gap at the base of the calcified nodule to loosen the connective tissue of the spongiosa layer, then the fibrosa layer at the calcified tissue could be completely peeled off easily (Fig. 1 and the Supplementary Video S1). This method ensures complete removal of the calcified tissue on the valve stump and the annulus. In the conventional group, calcified tissue on the valve stump and annulus was mechanically crushed by rongeur forceps, before the calcified debris was carefully removed. Once the diseased valve was excised and annulus debrided, a CarpentierEdwards Perimount Magna aortic pericardial valve (Edwards Lifesciences, Irvine, CA) was implanted with continuous or interrupted suturing. The traction sutures were removed and the aortotomy was closed. Once the patient was weaned from $\mathrm{CPB}$, transesophageal echocardiography was employed to assess effective orifice area (EOA), mean gradient, correct positioning of the prosthesis and possible paravalvular leak (PVL). A single mediastinal drainage tube was placed into the anterior mediastinum before the closure of chest.

\subsection{Assessment of baseline profile, follow-up, and outcomes}

Patients received warfarin for anticoagulation for 6 months after operation in accordance with published guideline $^{13}$ and were followed up for one year after operation.

The primary outcome included indexed EOA and mean gradient, which were assessed by hemodynamic performance at 30 days, 3 months, 6 months and 1 year after operation. 
Secondary outcomes were perioperative data and early clinical outcomes, included transfusion requirement, mechanical ventilation time, length of ICU and hospital stay, reoperation, PVL, perioperative comobidities, prosthesis-related mortality and all-cause mortality. Among them, major bleeding event was defined as blood loss of more than $500 \mathrm{ml}$ within 1 hour or $200 \mathrm{ml}$ per hour for 3 hours or more. Cardiac tamponade and degree of PVL were determined by echocardiography. Diagnosis of myocardial infarction was based on the elevation of high-sensitivity cardiac troponin $\mathrm{T}$ and creatine kinase isoenzyme, the onset of new pathologic Q-waves or new left bundle branch block, or the imaging evidence of new wall motion abnormality. ${ }^{14-15}$ The cerebrovascular accident was determined by CT or magnetic resonance imaging (MRI) of the head in the presence of new onset of neurological deficit. Diagnosis of endocarditis was based on the scientific statement from American Heart Association. ${ }^{16}$ Respiratory failure was defined as partial pressure of oxygen in arterial blood $\left(\mathrm{PaO}_{2}\right)<60 \mathrm{mmHg}$.

\subsection{Statistical analysis}

Sample size calculation was based on the preliminary outcome. In patients undergoing MIAVR with the conventional technique, the mean (SD) indexed EOA was $0.89(0.13) \mathrm{cm}^{2} / \mathrm{m}^{2}$, which was assessed by echocardiography at 30 days after operation. In patients assigned to the FLS group, we expected to achieve at least $20 \%$ increase of indexed EOA. For a 2 -sided $\alpha=0.05$ and power of $0.90,35$ patients in each group were required.

Database management and statistical analysis were performed using SPSS 25.0 software (IBM-SPSS Inc, Armonk, NY). Continuous variables were presented as mean and standard deviation, and categorical variables were shown as percentage and numbers. Continuous variables were compared using Student's t test for normally distributed data and the Mann-Whitney U test for skewed data. Categorical variables were compared by Chi-square or fisher exact test.

\section{RESULTS}

\subsection{Characteristics of the patients}

No significant differences in baseline characteristics were observed between the FLS group and the conventional group (Table 1). Figure 2 outlines the Consolidated Standards of Reporting Trials (CONSORT) diagram.

\subsection{Procedural outcomes}

In intention-to-treat analysis, a significant reduction in the cross-clamp time, CPB time and operative time was observed in the FLS group compared with the conventional group (Table 2). The ratio of continuous suture implantation of a bioprosthetic valve was also significantly higher in the FLS group than that in the conventional group $(27 / 30$ vs $5 / 30, \mathrm{p}<0.001)$. There was no significant difference in implanted valve size between two groups. In per-protocol analysis, the two groups showed comparable procedural outcomes .

\subsection{Perioperative and early clinical outcomes}

Significantly shorter ICU and hospital stay was observed in the FLS group, while other parameters were not statistically significant between the two groups. In per-protocol analysis, only hospital stay was significantly shorter in the FLS group compared with the conventional group (Table 3).

No myocardial infarctions, prosthesis endocarditis, respiratory failures, or deaths were observed in either group. One patient in the FLS group had mild PVL, whereas 4 patients in the conventional group had PVL (one mild, 2 moderate, and one severe). However, there was no significant difference in PVL incidence between the two groups, based on either in intention-to-treat $(\mathrm{P}=0.483)$ or per-protocol analysis $(\mathrm{P}=0.609)$. 2 patients in FLS and 6 patients in conventional groups experienced cerebrovascular events during perioperation. Although the number of cerebrovascular events was far more in the conventional group, there was no statistical difference between two groups. Cerebrovascular events progressed to permanent strokes in 2 patients from the conventional group, whereas the other 6 patients had no sequelae. 


\subsection{Hemodynamic performance}

In intention-to-treat analysis, we observed higher indexed EOA and lower mean gradient at all time points (30 days, 3 months, 6 months and 1 year after surgery) in the FLS group compared to the conventional group (Table 4). Per-protocol analysis showed comparable results between two groups.

\section{DISCUSSION}

To our knowledge, this is the first report of the FLS technique being applied in treating severe aortic valve calcification. Compared with the conventional technique, the FLS technique was associated with: (1) shorter cross-clamp time, CPB time and operative time, (2) shorter hospital stay, and (3) superior hemodynamic performance of the bioprosthetic aortic valves.

The incidence of calcific AS increases with age, with an average of $0.2 \%$ in patients of 50-59 years old and $9.8 \%$ in patients of $80-89$ years old. ${ }^{17}$ Calcific AS occurs 10-15 years earlier in population with congenital bicuspid aortic valves than normal controls. ${ }^{18}$ Calcification of the aortic valve is progressive and involves multiple mechanisms, including lipoprotein deposition, chronic inflammation, mineralization, osteoblastic transition of interstitial cells and active leaflet calcification. ${ }^{10-11}$ Pathological analysis of diseased valves revealed that fibrocalcific changes predominantly occur in the fibrosa layer and extend to the aortic side of the valve, which maybe associated with the spatial distribution of several anti-osteogenic genes expressed by endothelium. The endothelium covering the aortic side of leaflets shows lower expression of anti-osteogenic genes than that on the ventricular side. ${ }^{10}$ Based on these pathological characteristics of calcified aortic valve, we designed the new technique that thoroughly removes calcified tissue by stripping the fibrosa layer of the diseased valve and the annulus, namely, the FLS technique.

Our study showed that the FLS technique reduced cross-clamp time, CPB time and operation time in MIAVR. This can be attributed to two factors: (1) the FLS technique requires significantly shorter time to remove calcified tissue, (2) the FLS technique causes less damage to the aortic annulus and leaves less residual calcified tissue, which allows use of continuous sutures that takes less time. MIAVR offers several advantages over full sternotomy AVR, including reduced trauma and pain, shorter ICU and hospital stay, improved cosmesis, and lower rate of atrial fibrillation. However, the cross-clamp time and CPB time are prolonged in MIAVR than full sternotomy AVR, ${ }^{19-20}$ which is associated with increased mortality and mobility. ${ }^{21-22}$ By implementing the FLS technique, we have overcome these limitations and promoted the application of MIAVR in treating severe AS.

In current study, 1 patient had severe, 2 patients had moderate and 1 patient had mild PVL postoperation in conventional group but only 1 patient in FLS group had mild PVL. However, there was no significant difference between groups regarding the incidence and constituent ratio of the degree of PVL. Conventional methods of mechanically crushing calcified tissue can destroy the normal fibrous tissue in the annulus and reduce its strength and tensile force, which causes the annulus to be damaged by sutures. Moreover, calcified tissue in the aortic annulus and residual valve is also difficult to be completely cleared by conventional method, which may also contribute to PVL.

The prevalence of cerebrovascular events varies from $1 \%$ to $17 \%$ among patients with calcific AS who underwent AVR. ${ }^{23-24}$ Leker RR et.al reported that the risk factors for cerebrovascular events after AVR include longer bypass duration, older age, and larger pre-existing lesion burdens. ${ }^{25}$ Moreover, debris produced by mechanical crushing of calcified tissue is easy to fall into the left ventricle and hidden in intricate muscle bundles, which may enter the arterial system through left ventricular ejection and induce cerebro-arterial embolism. In this study, the incidence of cerebrovascular events and permanent strokes were $20.7 \%$ and $6.9 \%$ in conventional group in per-protocol analysis, while that in FLS group were $7.1 \%$ and 0 respectively. However, there was no differences between the two groups, probably due to the small number of enrolled patients and the fact that we only performed the neurological examinations on symptomatic patients, thus silent cerebral events may have been missed. ${ }^{26}$

Patients in FLS group obtained a significantly larger indexed EOA postoperatively than conventional group 
whether analyzed by intention-to-treat or per-protocol. The reason maybe that leaflets and annular calcification can be completely removed by FLS technology, so diameter and elasticity of aortic annular can be restored to normal state. Therefore, we can implant a aortic bioprothesis of optimal annulus size but not an undersized. However, small aortic root has remained a challenge. Small aortic root was observed in $17 \%$ of asymptomatic patients with mild to moderate AS, who had a significantly smaller annulus diameter in comparison with those with a normal aortic root. Enlargement is necessary to increase the indexed EOA during AVR for patients with small aortic annular. However, only $5 \%$ of patients received an annular enlargement procedure during AVR due to increased operative mortality and major morbidity. ${ }^{27}$ The residual calcification on aortic annulus or valve is the main cause for aborted annular enlargement during AVR, especially when the calcium extends down into the left ventricular outflow tract or the mitral valve. In this study, all patients with small aortic root in the FLS group received annular enlargement before implantation of a proper sizing of the prosthetic valve. In contrast, 2 patients with small aortic root in the conventional group did not receive concomitant annular enlargement due to the residual calcified tissue. As a result, oversized bioprostheses were implanted in order to avoid prosthesis-patient mismatch (PPM). Compared with annular enlargement, bioprosthesis oversizing can only increase a very limited valve size. In addition, oversizing alters aortic annular configuration and valvular hemodynamics, which leads to decreased EOA and increased pressure gradients. ${ }^{28}$ Therefore we conclude that using FLS technique in annular enlargement should be conducted on more patients with small aortic root in order to achieve better hemodynamic performance and patient outcome.

There remain several limitations in current study. First, it is a single-center trial with a small cohort size. Second, Patients are limited to bioprosthetic valve replacement, so most of them are frail elderly who have higher average age and more comobidities than the real world, which may cause selection bias. Third, all operations were performed by one surgeon who may have more experience with the FLS technique than the conventional technique. Fourth, we only followed up for one year, and long-term effect has not be established. Therefore, long-term safety and efficacy study of FLS in a larger cohort of patients is needed in the future.

\section{CONCULUSIONS}

Our study showed that the FLS technique is a feasible and effective method to remove calcified tissue in severe calcific AS. This innovative technique significantly reduces cross-clamp time and CPB time, thus minimizes the adverse effects of extracorporeal circulation and is associated with better hemodynamic performance. Our study advances the MIAVR technology and surgical treatment of severe calcific AS.

\section{FUNDING}

\section{None}

Conflict of interest: none declared.

\section{ACKNOWLEDGMENTS}

We are indebted to Yingjian Li for assistance with the data gathering and sorting.

\section{REFERENCES}

1 Cosgrove DM 3rd, Sabik JF. Minimally invasive approach for aortic valve operations. Ann Thorac Surg . 1996;62:596-597.

2 Ito T, Maekawa A, Hoshino S, et al. Right infraaxillary thoracotomy for minimally invasive aortic valve replacement. Ann Thorac Surg . 2013;96:715-717.

3 Yamazaki M, Kin H, Kitamoto S, et al. Efficacy of the stonehenge technique for minimally invasive aortic valve replacement via right infraaxillary thoracotomy. Ann Thorac Cardiovasc Surg . 2017;23:45-48.

4 Young CP, Sinha S, Vohra HA. Outcomes of minimally invasive aortic valve replacement surgery. Eur $J$ Cardiothorac Surg . 2018;53(suppl_2):ii19-ii23. 
5 Pope NH, Ailawadi G. Minimally invasive valve surgery. J Cardiovasc Transl Res . 2014;7:387-394.

6 Totsugawa T, Hiraoka A, Tamura K, et al. Ultrasonic annular debridement in minimally invasive aortic valve replacement. Gen Thorac Cardiovasc Surg . 2019.

7 Schwinger ME, Colvin S, Harty S, et al. Clinical evaluation of high-frequency (ultrasonic) mechanical debridement in the surgical treatment of calcific aortic stenosis. Am Heart J . 1990;120(6 Pt 1):1320-5.

8 Dweck MR, Boon NA, Newby DE. Calcific aortic stenosis: A disease of the valve and the myocardium. $J$ Am Coll Cardiol . 2012;60:1854-1863.

9 Sell S, Scully RE. Aging changes in the aortic and mitral valves. Histologic and histochemical studies, with observations on the pathogenesis of calcific aortic stenosis and calcification of the mitral annulus. Am J Pathol . 1965;46:345-365.

10 Leopold JA. Cellular mechanisms of aortic valve calcification. Circ Cardiovasc Interv . 2012;5:605-614.

11 Lindman BR, Clavel MA, Mathieu P, et al. Calcific aortic stenosis.Nat Rev Dis Primers . 2016;2:16006.

12 Joseph J, Naqvi SY, Giri J, et al. Aortic stenosis: Pathophysiology, diagnosis, and therapy. Am J Med . 2017;130:253-263.

13 Baumgartner H, Falk V, Bax JJ, De Bonis M, et al. 2017 esc/eacts guidelines for the management of valvular heart disease. Eur Heart J . 2017;38:2739-2791.

14 Cubero-Gallego H, Heredia-Rodriguez M, Tamayo E. Perioperative myocardial infarction after heart valve surgery, where are we going? J Thorac Cardiovasc Surg . 2018;155:1054-1055.

15 Cubero-Gallego H, Lorenzo M, Heredia M, et al. Diagnosis of perioperative myocardial infarction after heart valve surgery with new cut-off point of high-sensitivity troponin $t$ and new electrocardiogram or echocardiogram changes. J Thorac Cardiovasc Surg . 2017;154:895-903.

16 Baddour LM, Wilson WR, Bayer AS, et al. Infective endocarditis in adults: Diagnosis, antimicrobial therapy, and management of complications: A scientific statement for healthcare professionals from the american heart association. Circulation . 2015;132:1435-1486.

17 Eveborn GW, Schirmer H, Heggelund G, et al. The evolving epidemiology of valvular aortic stenosis. The tromso study. Heart . 2013;99:396-400.

18 Fishbein GA, Fishbein MC. Pathology of the aortic valve: Aortic valve stenosis/aortic regurgitation. Curr Cardiol Rep . 2019;21:81.

19 Chang C, Raza S, Altarabsheh SE, et al. Minimally invasive approaches to surgical aortic valve replacement: A meta-analysis. Ann Thorac Surg . 2018;106:1881-1889.

20 Phan K, Xie A, Di Eusanio M, et al. A meta-analysis of minimally invasive versus conventional sternotomy for aortic valve replacement.Ann Thorac Surg . 2014;98:1499-1511.

21 Iino K, Miyata H, Motomura N, et al. Prolonged cross-clamping during aortic valve replacement is an independent predictor of postoperative morbidity and mortality: Analysis of the japan cardiovascular surgery database. Ann Thorac Surg . 2017;103:602-609.

22 Madhavan S, Chan SP, Tan WC, et al. Cardiopulmonary bypass time: Every minute counts. J Cardiovasc Surg (Torino) . 2018;59:274-281.

23 Filsoufi F, Rahmanian PB, Castillo JG, et al. Incidence, imaging analysis, and early and late outcomes of stroke after cardiac valve operation. Am J Cardiol . 2008;101:1472-1478.

24 Messé SR, Acker MA, Kasner SE, et al. Stroke after aortic valve surgery: Results from a prospective cohort. Circulation . 2014;129:2253-2261. 
25 Leker RR, Messé SR, Erus G, et al. What makes new ischemic lesions symptomatic after aortic valve replacement? J Stroke Cerebrovasc Dis . 2017;26:2943-2948.

26 Floyd TF, Shah PN, Price CC, et al. Clinically silent cerebral ischemic events after cardiac surgery: Their incidence, regional vascular occurrence, and procedural dependence. Ann Thorac Surg . 2006;81:2160-2166.

27 Hawkins RB, Beller JP, Mehaffey JH, et al. Incremental risk of annular enlargement: A multi-institutional cohort study. Ann Thorac Surg . 2019;108:1752-1759.

28 Cleveland JD, Bowdish ME, Eberhardt CE, et al. Evaluation of hemodynamic performance of aortic valve bioprostheses in a model of oversizing. Ann Thorac Surg . 2017;103:1866-1876.
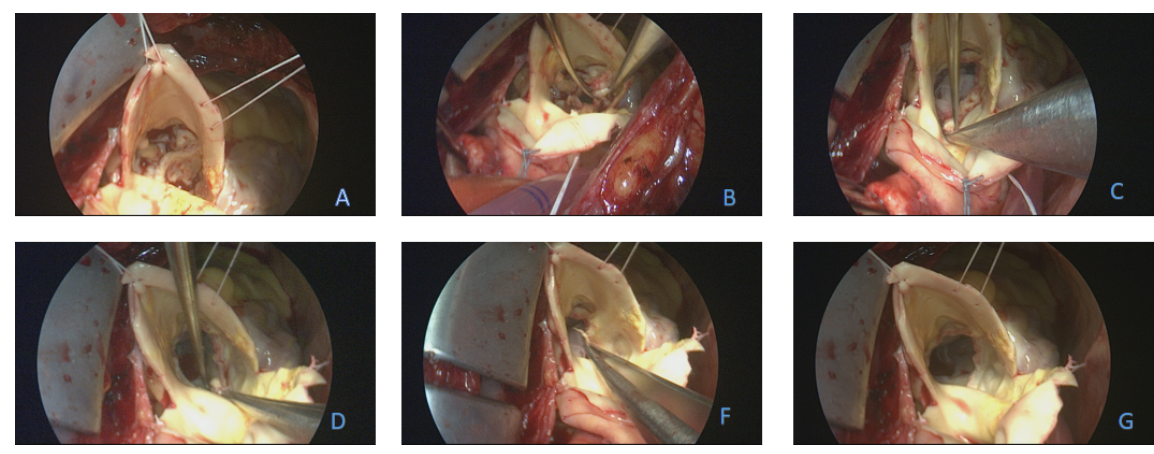


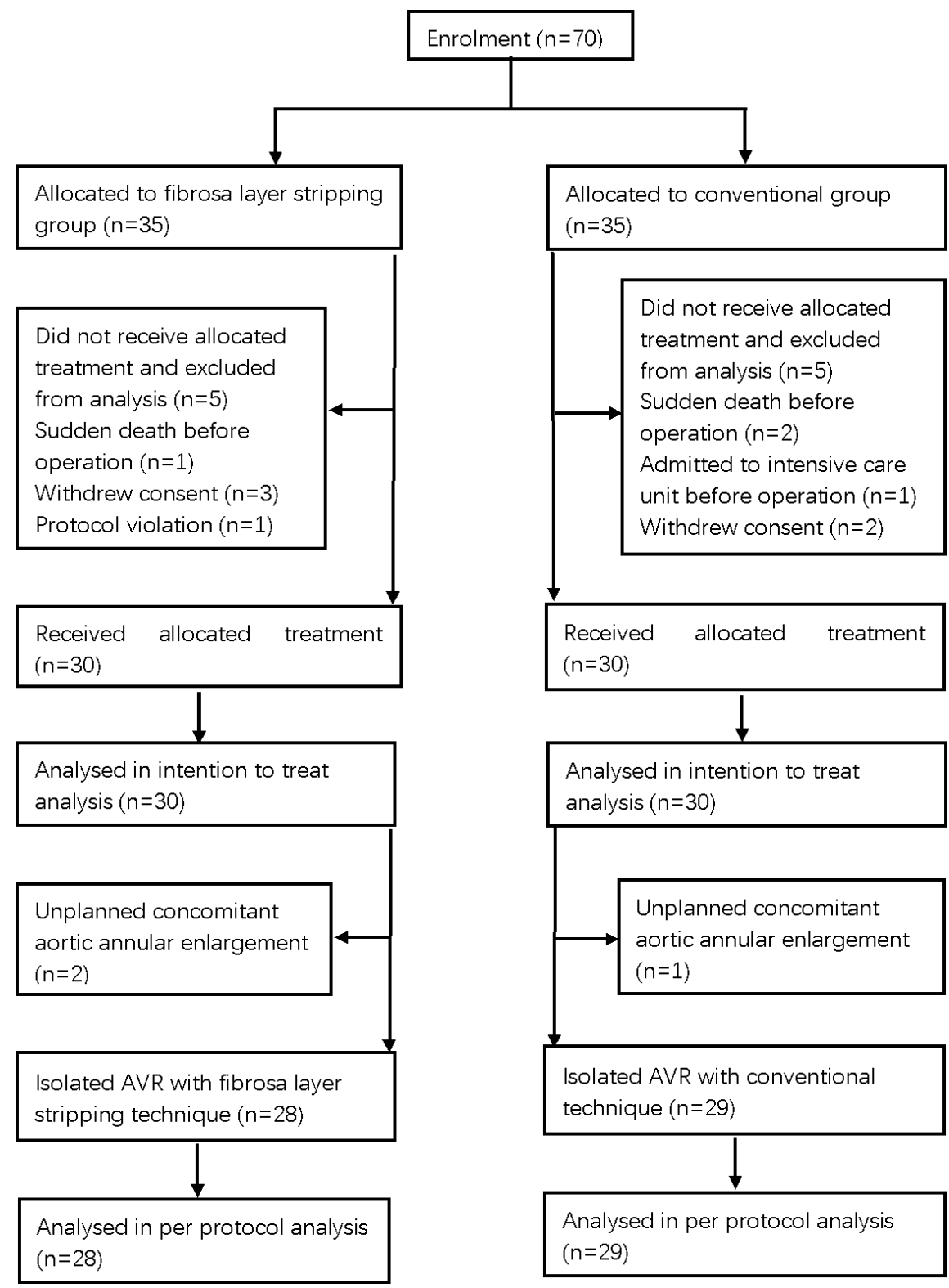

\section{Hosted file}

table 1.xls available at https://authorea.com/users/347884/articles/473395-the-benefit-offibrosa-layer-stripping-technique-during-minimally-invasive-aortic-valve-replacementfor-calcified-aortic-valve-stenosis-a-randomized-controlled-trial

\section{Hosted file}

table 2.xls available at https://authorea.com/users/347884/articles/473395-the-benefit-offibrosa-layer-stripping-technique-during-minimally-invasive-aortic-valve-replacementfor-calcified-aortic-valve-stenosis-a-randomized-controlled-trial

\section{Hosted file}

table 3.xls available at https://authorea.com/users/347884/articles/473395-the-benefit-of- 
fibrosa-layer-stripping-technique-during-minimally-invasive-aortic-valve-replacementfor-calcified-aortic-valve-stenosis-a-randomized-controlled-trial

\section{Hosted file}

table 4.xls available at https://authorea.com/users/347884/articles/473395-the-benefit-offibrosa-layer-stripping-technique-during-minimally-invasive-aortic-valve-replacementfor-calcified-aortic-valve-stenosis-a-randomized-controlled-trial 